Acta Crystallographica Section F

Structural Biology

and Crystallization

Communications

ISSN 1744-3091

Jeff Christensen, ${ }^{\text {a }}$ Cory J. Gerdts, ${ }^{b}$ Mathew C. Clifton ${ }^{b}$ and Lance Stewart ${ }^{\mathrm{b}}$

${ }^{a}$ Emerald BioStructures, 7869 NE Day Road West, Bainbridge Island, WA 98110, USA, and b Emerald BioSystems, 7869 NE Day Road West, Bainbridge Island, WA 98110, USA

Received 12 February 2011

Accepted 14 June 2011

\section{Salvage and storage of infectious disease protein targets in the SSGCID high-throughput crystallization pathway using microfluidics}

The MPCS Plug Maker is a microcapillary-based protein-crystallization system for generating diffraction-ready crystals from nanovolumes of protein. Crystallization screening using the Plug Maker was used as a salvage pathway for proteins that failed to crystallize during the initial observation period using the traditional sitting-drop vapor-diffusion method. Furthermore, the CrystalCards used to store the crystallization experiments set up by the Plug Maker are shown be a viable container for long-term storage of protein crystals without a discernable loss of diffraction quality with time. Use of the Plug Maker with SSGCID proteins is demonstrated to be an effective crystal-salvage and storage method.

\section{Salvaging protein targets}

The Seattle Structural Genomics Center for Infectious Disease (SSGCID) seeks to determine the molecular structures of bacterial proteins thought to be good targets for drug therapies, with a target of 75 structures per year over five years. These protein targets arrive at the crystallization core facility at Emerald BioStructures in highly purified and concentrated form as frozen aliquots. A schematic of the workflow used in this study is shown in Fig. 1. 10-15 new targets arrive at the facility each week and are immediately screened for crystallization using traditional sitting-drop vapor diffusion. Following the strategy proposed by Newman et al. (2005), all targets are screened against two 96-condition screens, JCSG+ (Emerald BioSystems) and PACT Premier (Molecular Dimensions), giving 192 unique conditions. The experiments are monitored for four weeks

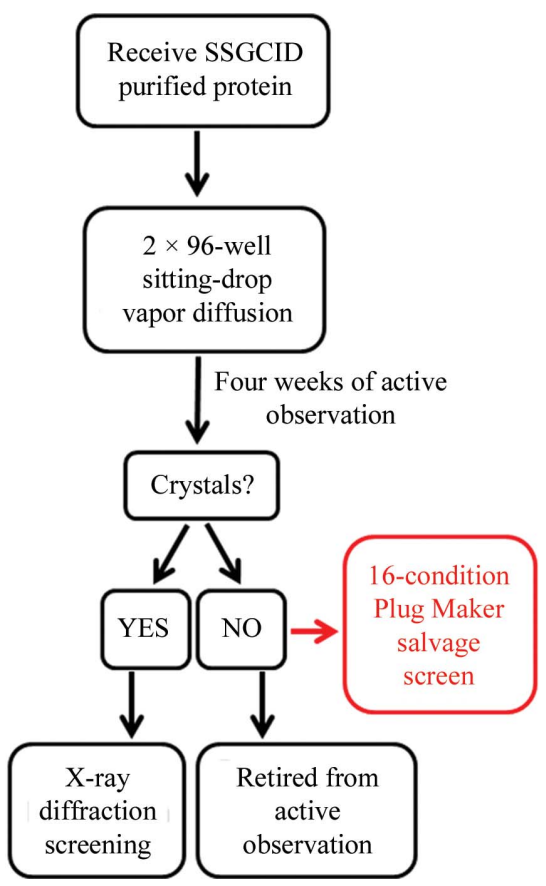

Figure 1

Workflow schematic for the salvage study. 
and any crystals that form enter an optimization pathway that includes testing for protein crystal diffraction, subsequent crystal optimization and structure determination. Inevitably, there are protein targets that produce no crystals within four weeks or fail in the optimization pathways. Typically, these targets are retired from active observation to open space for the next set of incoming targets. Within the Emerald BioStructures crystallization core facility, strategies have been utilized to attempt to salvage the structure determination of retired proteins. Such salvage pathways have included limited proteolysis (McPherson et al., 2004) and exogenous nucleation (D'Arcy et al., 2003; Thakur et al., 2007), although both had very limited success for the required effort involved.

With close to 900 retired protein targets currently available for salvage, the MPCS (Microcapillary Protein Crystallization System) Plug Maker (Emerald BioSystems) was used to thoroughly re-screen a small set of retired proteins. The plug-based microfluidic technology encompassed in the Plug Maker is able to thoroughly screen crystallization conditions by quickly and automatically generating concentration gradients for each condition that is screened. Details of the Plug Maker and its underlying technology have been published previously (Gerdts et al., 2008, 2010; Li et al., 2006; Zheng et al., 2003, 2005). Using this technology, as many as $80020 \mathrm{nl}$ crystallization experiments can be set up in one CrystalCard (Emerald BioSystems; Fig. 2) using as little as $5 \mu \mathrm{l}$ protein sample. Furthermore, although they only use up $\sim 10 \mathrm{nl}$ of protein each, the experiments are large enough to produce diffraction-ready crystals $(50-200 \mu \mathrm{m}$ per side)

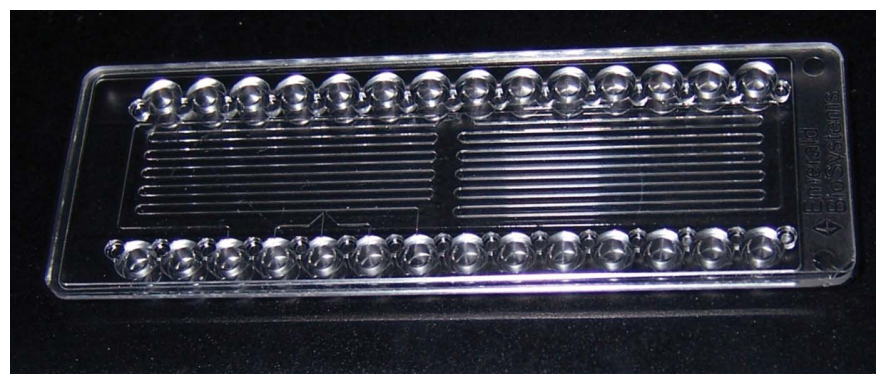

Figure 2

Image of a CrystalCard showing the two microcapillary channels.
Table 1

Concentrated conditions composing the MPCS screen (MPCS Sweet 16).

\begin{tabular}{|c|c|c|c|c|c|}
\hline $\begin{array}{l}\text { MPCS } \\
\text { Screen } \\
\text { No. }\end{array}$ & $\begin{array}{l}\text { Wizard } \\
\text { Full No. }\end{array}$ & JCSG+ & & At $100 \mathrm{~m} M$ & At $200 \mathrm{~m} M$ \\
\hline 1 & 1 & A-7 & $30 \%(w / v)$ PEG 8000 & CHES pH 9.5 & None \\
\hline 2 & 2 & E-3 & $20 \%(v / v)$ 2-propanol & HEPES pH 7.5 & $\mathrm{NaCl}$ \\
\hline 3 & 6 & A-2 & $30 \%(w / v)$ PEG 3000 & Citrate $\mathrm{pH} 5.5$ & None \\
\hline 4 & 9 & E-8 & $1.5 M\left(\mathrm{NH}_{4}\right)_{2} \mathrm{HPO}_{4}$ & Acetate $\mathrm{pH} 4.5$ & None \\
\hline 5 & 14 & E-1 & $1.4 \mathrm{M}$ sodium citrate & Cacodylate $\mathrm{pH} 6.5$ & None \\
\hline 6 & 17 & D-4 & $40 \%(w / v)$ PEG 8000 & Acetate $\mathrm{pH} 4.5$ & $\mathrm{Li}_{2} \mathrm{SO}_{4}$ \\
\hline 7 & 31 & $\mathrm{C}-1$ & $30 \%(w / v)$ PEG 8000 & Phosphate-citrate $\mathrm{pH} 4.2$ & $\mathrm{NaCl}$ \\
\hline 8 & 39 & A-6 & $30 \%(w / v)$ PEG 1000 & Phosphate-citrate $\mathrm{pH} 4.2$ & $\mathrm{Li}_{2} \mathrm{SO}_{4}$ \\
\hline 9 & 47 & E-4 & $2.52 \mathrm{M}\left(\mathrm{NH}_{4}\right)_{2} \mathrm{SO}_{4}$ & Tris $\mathrm{pH} 8.5$ & $\mathrm{Li}_{2} \mathrm{SO}_{4}$ \\
\hline 10 & 49 & $\mathrm{C}-7$ & $20 \%(w / v)$ PEG 3000 & Acetate $\mathrm{pH} 4.5$ & $\mathrm{Zn}(\mathrm{OAc})_{2}$ \\
\hline 11 & 51 & D-6 & $30 \%(w / v)$ PEG 8000 & Tris $\mathrm{pH} 8.5$ & $\mathrm{MgCl}_{2}$ \\
\hline 12 & 52 & E-2 & $3.0 M\left(\mathrm{NH}_{4}\right)_{2} \mathrm{SO}_{4}$ & Cacodylate $\mathrm{pH} 6.5$ & $\mathrm{NaCl}$ \\
\hline 13 & 59 & E-7 & $20 \%(v / v)$ 2-propanol & Cacodylate $\mathrm{pH} 6.5$ & $\mathrm{Zn}(\mathrm{OAc})_{2}$ \\
\hline 14 & 82 & E-12 & $20 \%(w / v)$ PEG 8000 & Imidazole $\mathrm{pH} 8.0$ & None \\
\hline 15 & 88 & E-6 & $30 \%(w / v)$ PEG 3000 & Imidazole $\mathrm{pH} 8.0$ & $\mathrm{Zn}(\mathrm{OAc})_{2}$ \\
\hline 16 & 91 & B-8 & $20 \%(w / v)$ PEG 8000 & Tris $\mathrm{pH} 7.0$ & $\mathrm{MgCl}_{2}$ \\
\hline
\end{tabular}

that can be examined in situ or extracted from the peel-apart CrystalCard (Gerdts et al., 2008, 2010; Yadav et al., 2005).

The JCSG+ screen used for the initial trials set up for SSGCID targets arose from data mining conducted by the Joint Center for Structural Genomics, which identified 96 conditions in commercial screens that had been most successful in crystallizing proteins (Newman et al., 2005; Page \& Stevens, 2004). 16 of these conditions were taken from Wizard Screens I and II (Emerald BioSystems) in order to compose the MPCS Sweet 16 Screen (Table 1).

Protein targets to be salvaged were screened against all or a portion of the MPCS Sweet 16 Screen based on the availability of remaining protein. No special criteria were used to select the targets other than that they were listed in our tracking spreadsheet as having failed to crystallize in either of the two standard vapor-diffusion trials during the initial four-week screening period. Subsequent to their selection for MPCS screening, the retired targets were re-examined in their original vapor-diffusion plates or selected for additional vapordiffusion trials for other reasons and ended up crystallizing sometime between two and six months after initial screening. These samples were still included in this analysis because the comparison to the
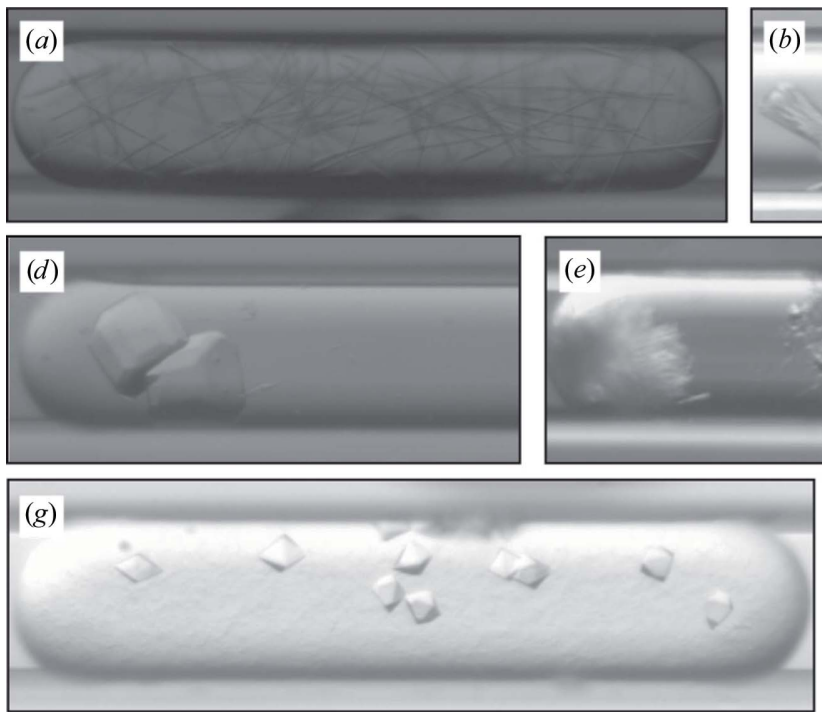
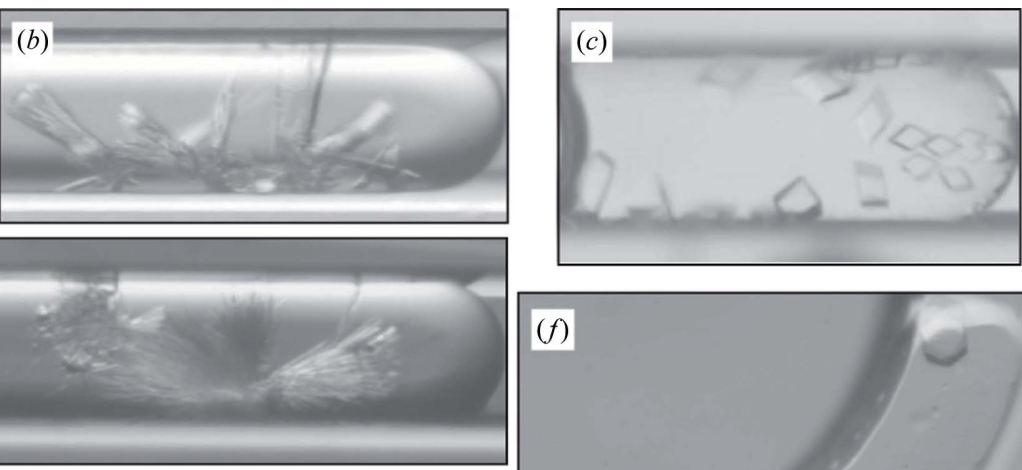

(h)

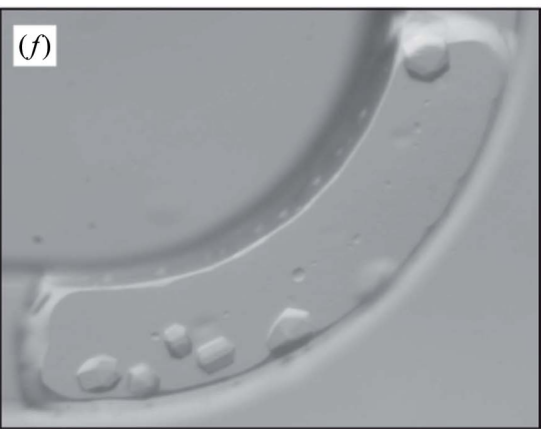

Figure 3

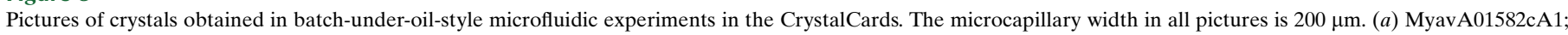
(b) MyavA01582aA1; (c) MyavA01566cA1; (d) MyavA00213aA1; (e) MysmA01566cA1; ( $f$ ) MyavA01582cA1; $(g)$ MythA00746aA1; $(h)$ GilaA01434aA1 
initial screening for retired targets remains valid (Table 3). One of the protein samples was not set up in vapor-diffusion trials because the volume delivered was inadequate and thus went directly to MPCS.

Crystallization experiments were set up in CrystalCards using the Plug Maker instrument. These experiments are analogous to batch crystallization, in which the crystallization reagents are combined in a drop (plug) surrounded by an oil and this initial condition is not expected to change significantly over time (see crystal pictures in Fig. 3). In this system, each plug is separated by a small volume of inert perfluorinated oil (Fluorinert FC-40, Sigma-Aldrich). The CrystalCard contains two separate microcapillaries and a thorough gradient of as many as 12 different crystallization conditions can be screened in each microcapillary, covering a wide range of crystallization phase space.

\section{Storing protein crystals}

Long-term storage of protein crystals at room temperature may be useful for the production of protein crystals for future research (ligand soaking) and may be useful owing to a lack of immediate

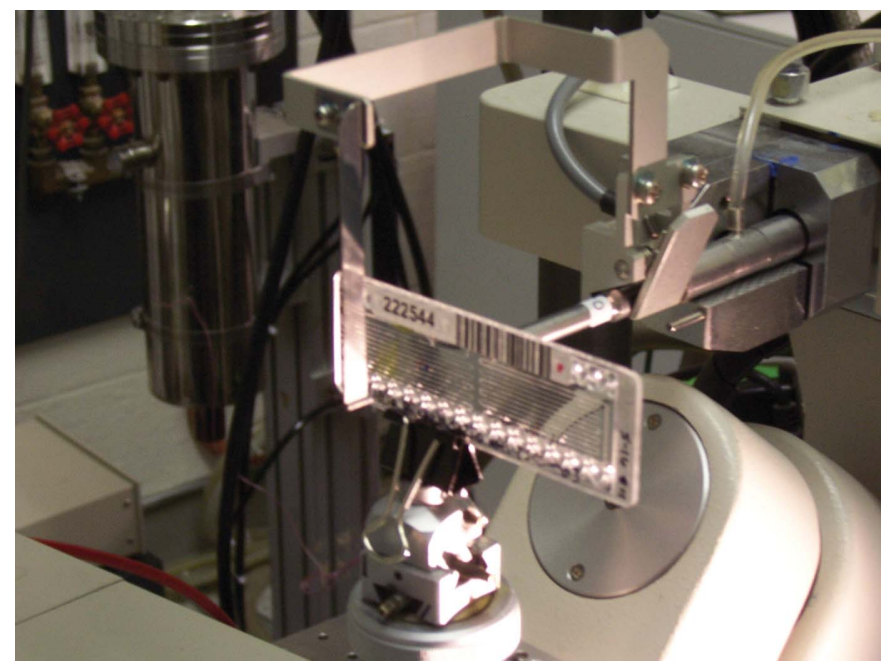

Figure 4

Picture of a CrystalCard mounted on a goniometer for in situ diffraction experiments.
Table 2

Data-collection statistics.

Values in parentheses are for the highest of 20 resolution shells

\begin{tabular}{lll}
\hline & MysmA.00391.bA1/3C & MysmA.01566.cA1 \\
\hline Space group & $P 2_{1}$ & $R 3 / R 3_{2}$ \\
Unit-cell parameters $\left(\AA{ }^{\circ}{ }^{\circ}\right)$ & $a=57.6, b=95.6$, & $a=b=87.8$, \\
& $c=115.3, \beta=99.6$ & $c=183.9$ \\
Wavelength $(\AA)$ & 1.5418 & 1.5418 \\
Resolution range $(\AA)$ & $50-2.8(2.90-2.80)$ & $50-2.6(2.69-2.60)$ \\
No. of unique reflections & $30844(3076)$ & $16285(1623)$ \\
Multiplicity & $3.5(3.4)$ & $2.7(2.7)$ \\
Completeness & $99.6(100)$ & $99.6(99.9)$ \\
$R_{\text {merge }} \dagger$ & $0.13(0.48)$ & $0.13(0.49)$ \\
Mean $I / \sigma(I)$ & $6.94(2.66)$ & $6.87(2.2)$ \\
\hline
\end{tabular}

$\dagger R_{\text {merge }}=\sum_{h} \sum_{i}\left|I_{i}(h)-\langle I(h)\rangle\right| / \sum_{h} \sum_{i} I_{i}(h)$.

access to X-ray data collection. Using traditional sitting-drop vapor diffusion or batch-under-oil techniques, protein-crystal storage can be difficult owing to evaporation of the crystallization drop. A long-term study of crystal stability in CrystalCards has shown that crystallization experiments inside CrystalCards can be stored without measurable evaporation and without meaningful loss of protein crystal diffraction quality. Crystals of BrabA.00113a.A1, an infectious disease target from SSCGID, were grown at the same time inside six CrystalCards and were subsequently examined using in situ X-ray diffraction by mounting the CrystalCard on the goniometer (Fig. 4; Gerdts et al., 2008; Yadav et al., 2005) periodically from April to December 2010. While not quantified, the crystals appeared to be unchanged and showed no obvious degradation of protein crystal diffraction quality over the course of eight months (Fig. 5). Furthermore, a $2.6 \AA$ resolution data set was collected from one of the protein targets salvaged in this study (MysmA00391bA1/3C) after the crystals had been stored in the CrystalCard for approximately five months (Table 2).

\section{Materials and methods}

\subsection{Crystallization screening conditions}

The similarity of microcapillary crystallization to batch crystallization makes it advantageous to start with crystallization solutions at higher component concentrations than are found in commercial sparse-matrix screens; thus, when running a concentration gradient of
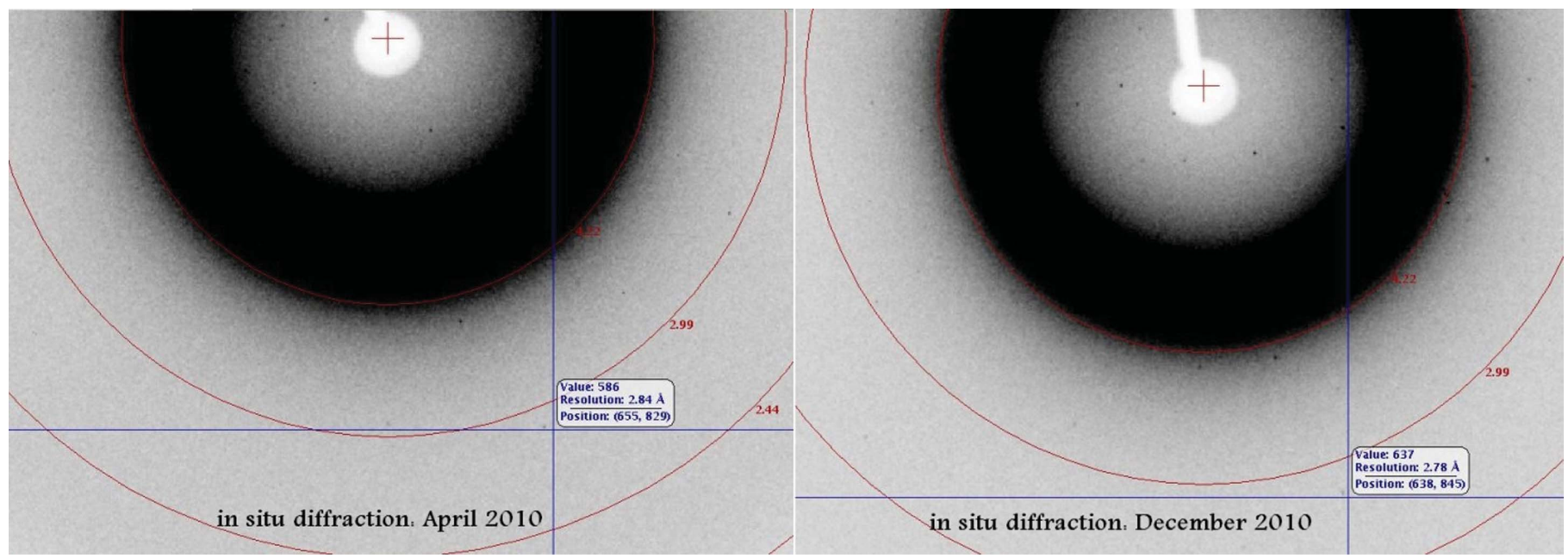

Figure 5

Comparison of in situ diffraction of BrabA.00113a.A1 cystals after long-term storage. Diffuse X-ray scatter is a result of the plastic material of the CrystalCard. 
Table 3

Summary of MPCS crystallization results.

\begin{tabular}{|c|c|c|c|c|c|c|c|}
\hline \multirow[b]{2}{*}{ SSGCID target protein } & \multirow[b]{2}{*}{ MPCS sample No. } & \multirow[b]{2}{*}{ MPCS crystals $\dagger$} & \multicolumn{4}{|c|}{ MPCS CrystalCard in situ diffraction screening and data collection } & \multirow{2}{*}{$\begin{array}{l}\text { Vapor-diffusion crystals } \\
\text { in retired trays } \S\end{array}$} \\
\hline & & & Protein diffraction $\ddagger$ & Likely protein & Not salt & Salt & \\
\hline AnphA.00481.aA1 & 1 & & & & & & \\
\hline AnphA.00462.aA1 & 2 & & & & & & \\
\hline BaheA.00227.aA1 & 3 & & & & & & \\
\hline BaheA.01161.aA1/3C & 4 & & & & & & \\
\hline EnhiA.01533.aA1 & 5 & $*$ & & $\mathrm{X}$ & & & \\
\hline GilaA.01434.aA1 & 6 & * & & $\mathrm{X}$ & & & \\
\hline LedoA.00425.aAG11 & 7 & * & & & * & & $*$ \\
\hline MyavA.00213.aA1 & 8 & $*$ & $\mathrm{XX}$ & & & & $*$ \\
\hline MyavA.00754.aA1 & 9 & $*$ & $\mathrm{XX}$ & & & & \\
\hline MyavA.00814.aA1 & 10 & & & & & & \\
\hline MyavA.00937.aA1 & 11 & $*$ & & $\mathrm{X}$ & & & $*$ \\
\hline MyavA.01097.hA1 & 12 & & & & & & \\
\hline MyavA.01097.iA1 & 13 & $*$ & & & $*$ & & $*$ \\
\hline MyavA.01188.aA1 & 14 & $*$ & & $\mathrm{X}$ & & & \\
\hline MyavA.01212.aA1 & 15 & $*$ & $\mathrm{XX}$ & & & & $*$ \\
\hline MyavA.01326.1A1 & 16 & & & & & & \\
\hline MyavA.01379.aA1 & 17 & $*$ & & $\mathrm{X}$ & & & $*$ \\
\hline MyavA.01530.cA1 & 18 & $*$ & & $\mathrm{X}$ & & & $*$ \\
\hline MyavA.01549.bA1 & 19 & & & & & & \\
\hline MyavA.01582.aA1 & 20 & * & $\mathrm{XX}$ & & & & PDB $3 q \mathrm{qj}$ \\
\hline MyavA.01582.cA1 & 21 & $*$ & $\mathrm{XX}$ & & & & $*$ \\
\hline MyavA.01625.aA1 & 22 & $*$ & & $\mathrm{X}$ & & & $*$ \\
\hline MyavA.01649.cA1 & 23 & $*$ & & $\mathrm{X}$ & & & \\
\hline MyavA.10520.bA1 & 24 & $*$ & & $\mathrm{X}$ & & & \\
\hline MyavA.17065.aA1 & 25 & & & & & & \\
\hline MyleA.00778.aA1 & 26 & $*$ & & $\mathrm{X}$ & & & $*$ \\
\hline MyleA.01155.aA1 & 27 & & & & & & \\
\hline MypaA.00249.aA1 & 28 & & & & & & \\
\hline MysmA.00337.bA1 & 29 & $*$ & & & & $*$ & \\
\hline MysmA.00391.bA1/3C & 30 & $*$ & $\mathrm{XXX}$ & & & & $*$ \\
\hline MysmA.01263.bA1 & 31 & * & & & & * & \\
\hline MysmA.01566.bA1 & 32 & * & & $\mathrm{X}$ & & & \\
\hline MysmA.01566.cA1 & 33 & * & $\mathrm{XXX}$ & & & & $*$ \\
\hline MythA.00746.aA1 & 34 & $*$ & $\mathrm{XX}$ & & & & $*$ \\
\hline Totals & 34 & 23 & 8 & 11 & 2 & 2 & 14 \\
\hline
\end{tabular}

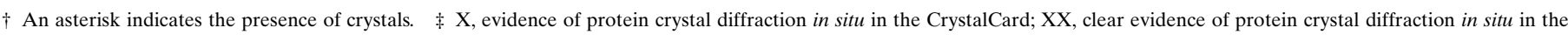
CrystalCard; XXX, complete X-ray diffraction data set collected from a crystal extracted from the peeled-apart CrystalCard. $\S$ An asterisk indicates the presence of crystals. A structure was deposited for MyavA.01582.aA1 with PDB code 3qlj.

a crystallization condition against a target protein sample the resulting plugs in the gradient will cover a wider crystallization space. During development of the MPCS Plug Maker system, custom versions of Wizard Screens I and II were designed in which the same reagents were used but at proportionally higher concentrations. Using the 16 conditions common to both the Wizard and JCSG+ screens would allow us to compare the microcapillary results with those of the initial vapor-diffusion trials; these conditions were dubbed the 'MPCS Sweet 16 Screen' (see Table 1).

\subsection{MPCS parameters}

The protein samples were set up using the Plug Maker hybrid protocol. Gradients of conditions $1-8$ and 9-16 of the Sweet 16 Screen were run in channels one and two, respectively. Approximately $5 \mu$ protein sample was required to screen all 16 conditions.

\subsection{CrystalCard storage}

CrystalCards were stored in standard plastic microscope slide boxes (VWR) with a small amount of water added to keep them humidified. The boxes were kept at $289 \mathrm{~K}$.

\subsection{Diffraction testing}

For in situ diffraction screening at room temperature, CrystalCards were mounted on the goniometer using a special clip (Fig. 4). The CrystalCards were oriented with the thin backing of the card facing the detector and the plug containing the crystal was aligned in the beam. Rotation of the card in the $\omega$ axis is limited to approximately $\pm 15^{\circ}$ from $0^{\circ}$ because of interference with the beam stop as well as the increasing thickness of the card that the X-ray beam must penetrate when the card is not perpendicular to the beam. A few $30 \mathrm{~s} / 0.5^{\circ}$ test images were collected within this range and examined for protein diffraction. If no obvious diffraction was noted a salt test was performed.

\subsection{Salt test}

With the card mounted and aligned as above, the detector was moved in to $50 \mathrm{~mm}$ and the $2 \theta$ axis moved out to $45^{\circ}$. A wedge of $1 \% / 10 \mathrm{~s}$ images at least $15^{\circ}$ wide was collected and if no small-molecule reflections were observed the crystal was characterized as 'not salt'.

\section{Results and discussion}

Microfluidic salvage efforts were attempted on 34 targets. 23 produced crystals or crystal-like objects. Through in situ X-ray diffraction screening eight of these showed obvious protein diffraction patterns, and complete data sets to $\sim 2.6 \AA$ resolution were collected for two targets from crystals harvested directly from the CrystalCards (Table 2). Because the crystals screened at room temperature may have sustained radiation damage, the data sets were collected at $100 \mathrm{~K}$ using different crystals from the same cards. Of the remaining crystals, 11 were characterized as 'likely protein' owing to a 
few very low-resolution reflections close to the beam stop, two were characterized as 'not salt' after failing the salt test (see §3) and two were confirmed as being salt crystals (Table 3).

The initial vapor-diffusion screening of SSGCID samples against only two 96-condition screens is minimal and has allowed the crystallization group to achieve the annual goals outlined in the goals of the SSGCID. Screening these same samples against a larger number of sparse-matrix screens may have resulted in crystals for a larger percentage of samples, but would come with the additional burden of more trials to set up, monitor and store, and a greater cost of consumables. Using the capabilities of the Plug Maker to screen a wide variety of concentrations of just 16 crystallization screening conditions has resulted in two high-resolution data sets and legitimate crystallization leads for many previously retired targets. While all of the vapor-diffusion crystals reported here appeared after four weeks, many of the MPCS crystals appeared in the CrystalCards within days.

The results of these preliminary MPCS experiments are very encouraging. Although nominally using a screen of only 16 conditions, the actual number of unique conditions is the number of plugs in the CrystalCard (up to 800) because each crystallization solution is run as a gradient. The ability to obtain crystallization leads, protein crystal diffraction and full diffraction data sets using less than $10 \mu \mathrm{l}$ protein sample, and with a success rate comparable to standard crystallization methods, represents a large saving in effort and indicates that efficient microfluidic scanning of protein and crystallization condition concentrations in initial crystallization trials using the MPCS Plug Maker can be a highly effective means of producing protein crystals.

In addition to soluble proteins, the Plug Maker may prove to be useful for crystallizing nucleic acids and membrane proteins, both of which represent special challenges. While preliminary experiments have shown that membrane proteins solubilized with some detergents form plugs normally, neither nucleic acids nor membrane proteins have been tested extensively by us. Experiments are planned to more thoroughly investigate this and the results of these experiments will be reported in due course.

The authors thank the entire SSGCID team. This research was funded under Federal Contract No. HHSN272200700057C from the National Institute of Allergy and Infectious Diseases, National Institutes of Health, Department of Health and Human Services. This work was also supported by the PSI-2 Specialized Center Grant U54 GM074961, co-sponsored by NIGMS-NCRR for the Accelerated Technologies Center for Gene to Three-Dimensional Structure. All protein targets were provided by the NIAID-supported Seattle Structural Genomics Center for Infectious Disease.

\section{References}

D'Arcy, A., Mac Sweeney, A. \& Haber, A. (2003). Acta Cryst. D59, 1343-1346. Gerdts, C. J., Elliott, M., Lovell, S., Mixon, M. B., Napuli, A. J., Staker, B. L., Nollert, P. \& Stewart, L. (2008). Acta Cryst. D64, 1116-1122.

Gerdts, C. J., Stahl, G. L., Napuli, A., Staker, B., Abendroth, J., Edwards, T. E., Myler, P., Van Voorhis, W., Nollert, P. \& Stewart, L. J. (2010). J. Appl. Cryst. 43, 1078-1083.

Li, L., Mustafi, D., Fu, Q., Tereshko, V., Chen, D. L., Tice, J. D. \& Ismagilov, R. F. (2006). Proc. Natl Acad. Sci. USA, 103, 19243-19248.

McPherson, A., Day, J. \& Harris, L. J. (2004). Acta Cryst. D60, 872-877.

Newman, J., Egan, D., Walter, T. S., Meged, R., Berry, I., Ben Jelloul, M., Sussman, J. L., Stuart, D. I. \& Perrakis, A. (2005). Acta Cryst. D61, 14261431.

Page, R. \& Stevens, R. C. (2004). Methods, 34, 373-389.

Thakur, A. S., Robin, G., Guncar, G., Saunders, N. F., Newman, J., Martin, J. L. \& Kobe, B. (2007). PLoS One, 2, e1091.

Yadav, M. K., Gerdts, C. J., Sanishvili, R., Smith, W. W., Roach, L. S., Ismagilov, R. F., Kuhn, P. \& Stevens, R. C. (2005). J. Appl. Cryst. 38, 900-905.

Zheng, B., Gerdts, C. J. \& Ismagilov, R. F. (2005). Curr. Opin. Struct. Biol. 15, $548-555$.

Zheng, B., Roach, L. S. \& Ismagilov, R. F. (2003). J. Am. Chem. Soc. 125, 11170-11171. 